\title{
A concept for the development of a pioneer regional Out-of-Hospital Cardiac Arrest Program to improve patient outcomes
}

\author{
Maciej Sip ${ }^{1,2}$, Mateusz Puślecki1,2,3, Tomasz Kłosiewicz 1,2, Radosław Zalewski1,2, Marek Dąbrowski2,4, Marcin Ligowski \\ Ewa Goszczyńska ${ }^{3}$, Christopher Paprocki², Marek Grygier ${ }^{5}$, Maciej Lesiak ${ }^{5}$, Marek Jemielity ${ }^{3}$, Bartłomiej Perek ${ }^{3}$ \\ 1 Department of Medical Rescue, Poznań University of Medical Sciences, Poznań, Poland \\ 2 Polish Society of Medical Simulation, Słupca, Poland \\ 3 Department of Cardiac Surgery and Transplantology, Poznań University of Medical Sciences, Poznań, Poland \\ 4 Department of Medical Education, Poznań University of Medical Sciences, Poznań, Poland \\ 5 1st Department of Cardiology, Poznań University of Medical Sciences, Poznań, Poland
}

\section{KEY WORDS}

basic life support, extended cardiopulmonary resuscitation, outcomes, out-of-hospital cardiac arrest

\section{ABSTRACT}

BACKGROUND Immediate initiation of cardiopulmonary resuscitation (CPR) increases chances of restoring spontaneous circulation and survival after out-of-hospital cardiac arrest (OHCA). For some refractory cases, extended cardiopulmonary resuscitation (ECPR) may be a promising option.

AIMS The aim of the study was to estimate the possibility of implementation of ECPR procedure to improve current early outcomes of patients after OHCA.

METHODS The medical charts of the Province Emergency Station in Poznań from a 12-month period were assessed retrospectively. All OHCA cases were identified and the following potential inclusion criteria for ECPR were analyzed: initial defibrillation rhythm, age between 18 and 65 years, CPR conducted by bystanders, and time to hospital arrival less than 40 minutes.

RESULTS In 576 (46.7\%) of 1233 identified OHCA cases, CPR was initiated by bystanders and automated external defibrillator was applied only 17 times. An initial defibrillation rhythm was noted in 138 individuals (11.2\%). Out of 65 patients who met the ECPR age criterion, 55 underwent CPR by bystanders which lead to a no-flow time that did not exceed 10 minutes. The additional 9 of them would be excluded due to time to hospital arrival. This means that ECPR would be applicable in 46 patients after OHCA.

CONCLUSIONS Our analysis showed that in some patients after sudden cardiac arrest, it would have been possible to implement ECPR as a crucial part of the Regional Out-of-Hospital Cardiac Arrest (OHCA) Program, and in consequence, probably to improve early outcomes of patients with refractory and potentially reversible cardiac arrest.

\section{Correspondence to:}

Maciej Sip, MSc, Department of Medical Rescue, Poznań University of Medical Sciences, ul. Rokietnicka 7, 60-806 Poznań, Poland, phone: +48504468364, email: msip@ump.edu.pl Received: April 11, 2020. Revision accepted: June 5, 2020 Published online: June 16, 2020. Kardiol Pol. 2020; 78 (9): 875-881 doi:10.33963/KP.15433 Copyright by the Author(s), 2020
INTRODUCTION According to the Statistics Poland, out of approximately 388000 deaths in 2016 (202 000 men [52.1\%] and 186000 women [47.9\%]), 43.7\% were due to cardiovascular reasons, mainly heart diseases $(60.6 \%)$ followed by cerebrovascular diseases (17.3\%)., ${ }^{1,2}$

In Europe, out-of-hospital cardiac arrest (OHCA) has the incidence of 350000 to 700000 (mean 500000 ) events per year, which gives an average of 55 to 113 events per 100000 inhabitants. ${ }^{3}$ The overall survival rate in OHCA is only $2 \%$ to $11 \%$ and is characterized by regional variability. ${ }^{4}$ In the best case scenario, in witnessed sudden cardiac arrest (SCA) with an initial shockable rhythm followed by early defibrillation and effective cardiopulmonary resuscitation (CPR), the return of spontaneous circulation (ROSC) may be achieved even in up to $30 \%$ or $40 \%$ of cases. ${ }^{5,6}$ Additionally, the use of an automated external defibrillator (AED) has a marked 


\section{WHAT'S NEW?}

Support of critically ill patients with underlying potentially reversible causes by means of extracorporeal membrane oxygenation (ECMO) has recently gained increased recognition. Poor early outcomes of patients after out-of-hospital cardiac arrest (OHCA) and our current experience in ECMO application encouraged us to implement ECMO in that cohort and to design the first study in Poland. Thus, the purpose of the present study was to estimate retrospectively the potential for extended cardiopulmonary resuscitation (ECPR) with ECMO on the base of the current outcomes of patients after OHCA. We found the ECPR would be applicable in 46 of them in the Wielkopolska Province in Poland.

positive impact on ROSC rate, particularly if it is applied within the first minutes of CPR., ${ }^{7,8}$

In patients after OHCA refractory to high-quality CPR, extended cardiopulmonary resuscitation (ECPR) may be considered. It was demonstrated to improve blood flow and oxygenation, thus preventing irreversible damage to organs and cerebral ischemia during SCA. ${ }^{9}$ A favorable impact of ECPR is evident if extracorporeal techniques are applied quickly (ideally within 1 hour of SCA) and when cardiac arrest results from potentially reversible causes. ${ }^{10}$ In these cases, ECPR gives time for and enables cardiovascular interventions such as percutaneous coronary angioplasty (in acute coronary syndrome) or thrombectomy (in acute pulmonary embolism). ${ }^{3}$ The proper use of ECPR in OHCA resistant to standard resuscitation techniques significantly increases the chances of patients' survival and even up to $1 / 4$ of those patients can be discharged with good neurological status. ${ }^{11}$

The aim of this study was to estimate the possibility of implementation of ECRP procedure with extracorporeal life support techniques as a potential background for the implementation of the pioneer Poznan Out-of-Hospital Cardiac Arrest Program (POHCA) $)^{12,13}$ to improve early outcomes of patients after OHCA.

METHODS Analysis of the current situation The medical charts of the Province Emergency Station in Poznań including all cases of SCA between March 1, 2018 and February 28, 2019 were analyzed. The dispatcher reports filled by the chief medical dispatcher after each incident of SCA were verified. Each report included data from the patient emergency card, that is, information obtained by the receiving dispatcher from the requesting person. The second part of the reports were clinical details collected by emergency medical service (EMS) paramedics who were at the site of every emergency. Data were collected using forms compatible with an international standard (Utstein), filled in by EMS teams immediately after the resuscitation or transfer to the hospital. Return of spontaneous circulation was defined in cases when a given patient with OHCA had successful
CPR on the site of emergency and was admitted alive to the emergency department of the nearest hospital.

Stratification of potential candidates for extended cardiopulmonary resuscitation The ECPR procedure designed previously by the team from the regional ECMO for Greater Poland program was adopted as a starting point for further considerations. ${ }^{14,15}$ Inclusion criteria for the potential ECPR were established according to the recommendations of the Extracorporeal Life Support Organization, based on the previous experience of the European Extracorporeal Life Support Organization Centers of Excellence. ${ }^{16,17}$ The analysis included the SCA reports of individuals whose initial heart rhythm, determined by EMS paramedics, was either ventricular fibrillation (VF) or pulseless ventricular tachycardia (pVT). Furthermore, patients younger than 18 and older than 65 years were excluded from the selected group, followed by those who were not resuscitated by bystanders for 10 minutes after SCA. Eventually, we estimated the transport time from the site of SCA to the Clinical Hospital of Lord's Transfiguration in Poznan, the referential place for implementation of the extracorporeal membrane oxygenation (ECMO) system, which is prepared for a full spectrum of emergent intravascular cardiac interventions. The tool used to determine the above mentioned data was the Google Maps application and was based on the shortest route and the average daily transport time.

Summarizing, the inclusion criteria in adherence to the ECPR protocol (with Ulstein protocol points provided in brackets) were as follows: 1 ) initial rhythm VF / pVT (first monitored rhythm, shockable); 2) age older than 18 and younger than 65 years (age, estimated); 3) bystanders CPR or less than 10 minutes to EMS (location of arrest, out of hospital + arrest witnessed + CPR before EMS arrival); transportation time to referral center less than 40 minutes (N/A).

Statistical analysis Data from the reports were uploaded to spreadsheets in Excel (Excel version 2016, by Microsoft Office). First, the Shapiro-Wilk test was used to assess whether variables (for example, transport time to site, the estimated time to the center of reference) satisfied the criteria for normal distribution. Data with normal distribution are presented as mean (SD) and others as median with range (minimum value-maximum value). Statistical analysis was performed using the Statistica 7.0 software package (Stat Soft Inc., Tulsa, Oklahoma, United States).

Ethical statement According to the rules of the local bioethical committee of Poznań University of Medical Sciences, the statement of ethics 
approval is not required for a retrospective data analysis of blinded patient documentation.

RESULTS In the 12-month period, EMS teams in the Poznań Metropolitan region were sent out 73100 times, including 1233 (1.7\%) cases qualified as OHCA (120 SCA per 100000 inhabitants). Most often, the remarks of the witnesses regarding patients status were as follows: "unconscious but breathing," "unconscious but breathing heavily," or "shortness of breath." In this group of 1233 cases, 455 reporting persons (36.9\%) did not recognize the cessation of basic vital functions and thus did not initiate any aspect of CPR (FIGURE 1). Only in 152 cases (12.3\%), the EMS dispatcher gave the instructions on how to perform basic life support (BLS).

The EMS notification patient status was "cardiac arrest" in $63.1 \%$ (confirmed by EMS) and "other" in $36.9 \%$ of all OHCA cases. In the latter subgroup ( $\mathrm{n}=455)$, OHCA was diagnosed by the paramedics, including 65 with SCA after paramedics arrival the site of the emergency.

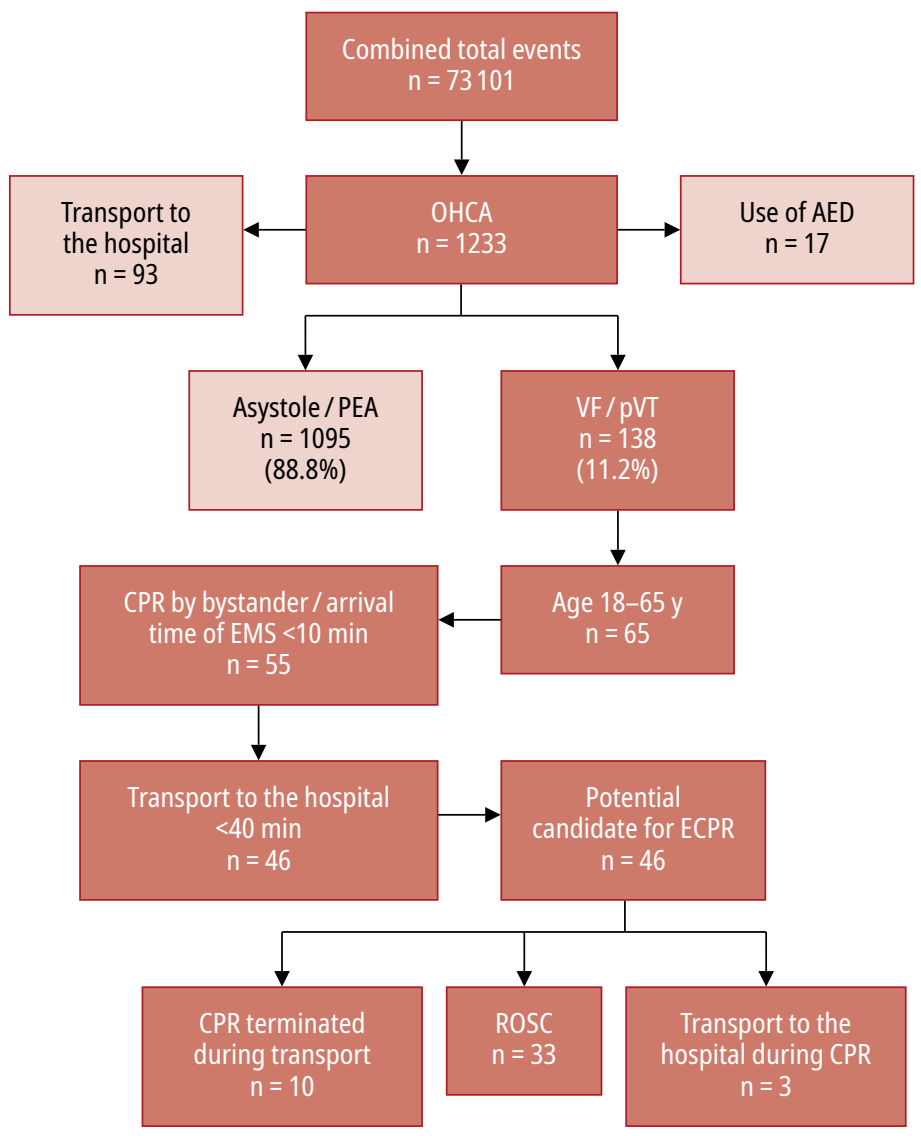

FIGURE 1 Stages of exclusion: from all included patients to potential candidates for extended cardiopulmonary resuscitation (ECPR)

Abbreviations: AED, automated external defibrillation; CPR, cardiopulmonary resuscitation;

ECPR, extended cardiopulmonary resuscitation; EMS, emergency medical service;

OHCA, outof-hospital cardiac arrest; PEA, pulseless electrical activity; ROSC, return of spontaneous circulation; VF/pVT, ventricular fibrillation / pulseless ventricular tachycardia
Site of notification The median (range) EMS arrival time to the site of emergency was 7 (2-24) minutes. Bystanders initiated CPR in 576 cases (46.7\%) and AEDs were applied in only 17 out of those cases (1.3\%). AEDs were used 14 times by the firefighters of the Polish State Fire Service and the Volunteer Fire Department, 1 time in a hotel, 1 time a court building, and 1 time by the ambulance team during a sporting event.

Out of 1233 patients with OHCA, asystole or pulseless electrical activity (PEA) was diagnosed as the initial rhythm in 1095 individuals (88.8\%), whereas $\mathrm{VF} / \mathrm{pVT}$ was diagnosed in the remaining 138 (11.2\%). In 564 patients (45.7\%), paramedics decided to continue or initiate advanced life support (ALS) (VF/pVT in 138 [24.5\%] out of 564 on-site CPR cases). In 67 cases ( $11.9 \%$ of on-site EMS resuscitations), ROSC was obtained. Out of those patients, 61 had VF / pVT and 6 had PEA at the time of EMS team arrival. Out of all patients with OHCA, 93 (7.5\%) were transported to the hospital, including 67 with ROSC and another 26 with ongoing CPR. The latter experienced recurrence of SCA (with ROSC noted during ALS; temporary ROSC) and were transported to the hospital with the support of a mechanical automated chest compression (MACC) device.

Presumptive candidates for extended cardiopulmonary resuscitation The adopted age criterion (18-65 years) for presumptive ECPR was met by only 65 patients, including 55 men (85\%) and 10 women (15\%). Bystanders performed CPR in 55 out of 65 individuals before the arrival of the EMS team, or if they did not perform CPD, the arrival time of paramedics did not exceed 10 minutes.

Potential transporting of patients with out-of-hospital cardiac arrest to the hospital Currently, the presumptive ECPR referential hospital is not the first choice for emergency cases, thus, only the minority of those cases were referred to that institution. The potential median (range) transport time from the site of the emergency to the referential hospital was estimated to be 13 (5-40) minutes. Applying this criterion of ECPR applicability, another 9 cases should have been excluded.

The fate of potential patients with out-of-hospital cardiac arrest for extended cardiopulmonary resuscitation A total of 46 patients with OHCA (3.7\% of the total number and $8.2 \%$ of the ALS group) met the inclusion criteria as described above and were potential candidates for ECPR.

The consecutive inclusion criteria in adherence to ECPR protocol (with the number of patients with OHCA included in the parentheses) are as follows: 1) initial rhythm VF / pVT (138); 2) age older than 18 and younger than 65 years 
(65); 3) bystanders CPR or less than 10 minutes to EMS arrival (55); 4) transportation time to referral center less than 40 minutes (46).

In reality, CPR had to be terminated in 10 cases prior to hospital admission. However, if the ECPR procedure had been available, these patients would probably have been referred as potential candidates and transported to the hospital.

The remaining 36 patients with $\mathrm{OHCA}$ were successfully transported to the hospital. Data for 10 of them are missing. Cardiac causes of SCA were noted in 22 of them, 17 underwent cardiac catheterization, and 14 had successful percutaneous coronary intervention with stent implantation. In another 3, pulmonary embolism was diagnosed. In the rest of the patients, decompensated congestive heart failure with severely impaired left ventricular systolic performance was detected. Probably life-threatening ventricular arrhythmias caused OHCA. Out of 36 patients after OHCA, 15 survived and were discharged but only 5 presented good neurological condition.

DISCUSSION The concept of the Poznan Out-of-Hospital Cardiac Arrest (POHCA) program came as a continuation of the ECMO for Greater Poland, a regional program established in 2016. ${ }^{13,18}$ The premise for initiating the program in Poland is the widespread implementation of ECMO devices as mechanical circulatory and respiratory support in potentially reversible critical conditions. Although there are known cases of ECPR implementation as a therapeutic option for patients with in-hospital cardiac arrest in Poland, there are no reports dealing with possible ECMO application in patients after OHCA.

The analysis of the EMS data revealed that the strategy of OHCA treatment in our region needed marked improvement. The first weak point of the current management with SCA was lack of fundamental knowledge of SCA and BLS maneuvers in the society. It needs to be emphasized that BLS is not reserved for medical professionals since the majority of OHCA witnesses (usually more than three-fourth) are usually bystanders. ${ }^{19}$ According to the available EMS charts analyzed by our team, more than half of OHCA witnesses did not undertake any resuscitative actions before the paramedics' arrival because they failed to identify SCA. In nearly $37 \%$ of cases, the bystanders did not report life-threatening symptoms to the chief medical dispatcher which obviously resulted in a significant delay in EMS reaction but also in a very low rate (approximately 12\%) of information provided to OHCA witnesses by EMS staff on how to perform CPR. This rate is markedly lower than in the earlier studies and it could have an impact on resuscitation prior to ambulance arrival. ${ }^{5}$ Moreover, such a small number of dispatcher instructions could be the reason that in our group of OHCA cases, the first shockable rhythms were noted less frequently than previously published. ${ }^{20,21}$ Our observations support earlier reports regarding the high rate of inappropriate SCA identification in the Polish population. Even students of nonmedical faculties were previously found to present inadequate knowledge and skills in BLS. ${ }^{22}$

The Public Access to Defibrillation (PAD) program was launched many years ago to increase chances of survival of OHCA..$^{23}$ Its goal was to place AEDs in the areas where SCA had occurred at least once in the last 2 years or where a rescue team could not arrive within 5 minutes. ${ }^{24}$ This initiative was absolutely right as the studies have shown the importance of the time between SCA and the correct use of an AED. If the time does not exceed 3 minutes, OHCA survival can be increased by up to $70 \% .{ }^{3} \mathrm{~A}$ significant increase in the survival rate of OHCA was confirmed after effective implementation of the PAD program in some countries. ${ }^{24}$ Unfortunately, in our region, despite the increasing number of AEDs (26 registered in 2018), our study showed a dramatically low rate (roughly only 1\%) of their use. Again, the reason for this is lack of knowledge. ${ }^{25}$ Keeping in mind the fact that the rhythm initiating cardiac arrest is usually a defibrillation one $(\mathrm{VF} / \mathrm{pVT}),{ }^{11}$ and that every minute of delay in initiating CPR rapidly decreases the chances for ROSC, BLS education of the Polish society should be a priority for healthcare providers.

The presence of an initial VF / pVT rhythm (detected by the paramedics at the time of arrival) in $94 \%$ of patients confirms a no-flow time of less than 10 minutes. ${ }^{26}$ Asystole/PEA as an initial rhythm is regarded as a negative prognostic factor. Although PEA may occur as an etiology of the reversible causes of cardiac arrest, patients in asystole / PEA are not assumed to be candidates for ECPR. ${ }^{27,28}$ The latter statement was supported in a study by Nakashima et $\mathrm{al}^{29}$ in which the best 30-day survival rate was shown after VF / pVT with ECPR (40\%), followed by PEA/asystole with ECPR (16\%), VF/pVT with conventional CPR (10\%), and asystole / PEA with conventional CPR (only 4\%). 17,18,29

Extended cardiopulmonary resuscitation as an invasive and expensive method is not one for all patients even in the wealthiest countries. A significant correlation between age, probability of survival, and good neurological outcome was found. It was noted that the success rate of ECMO is significantly reduced in patients over 65 years of age. ${ }^{30}$ However, active ECMO centers that successfully implemented ECPR programs accept a wide age range of patients, with a lower limit of 18 and an upper of 65 (Melbourne, Prague), 70 (Paris), and even 75 years of age (Taipei, Minneapolis). 17,31-33 In our 


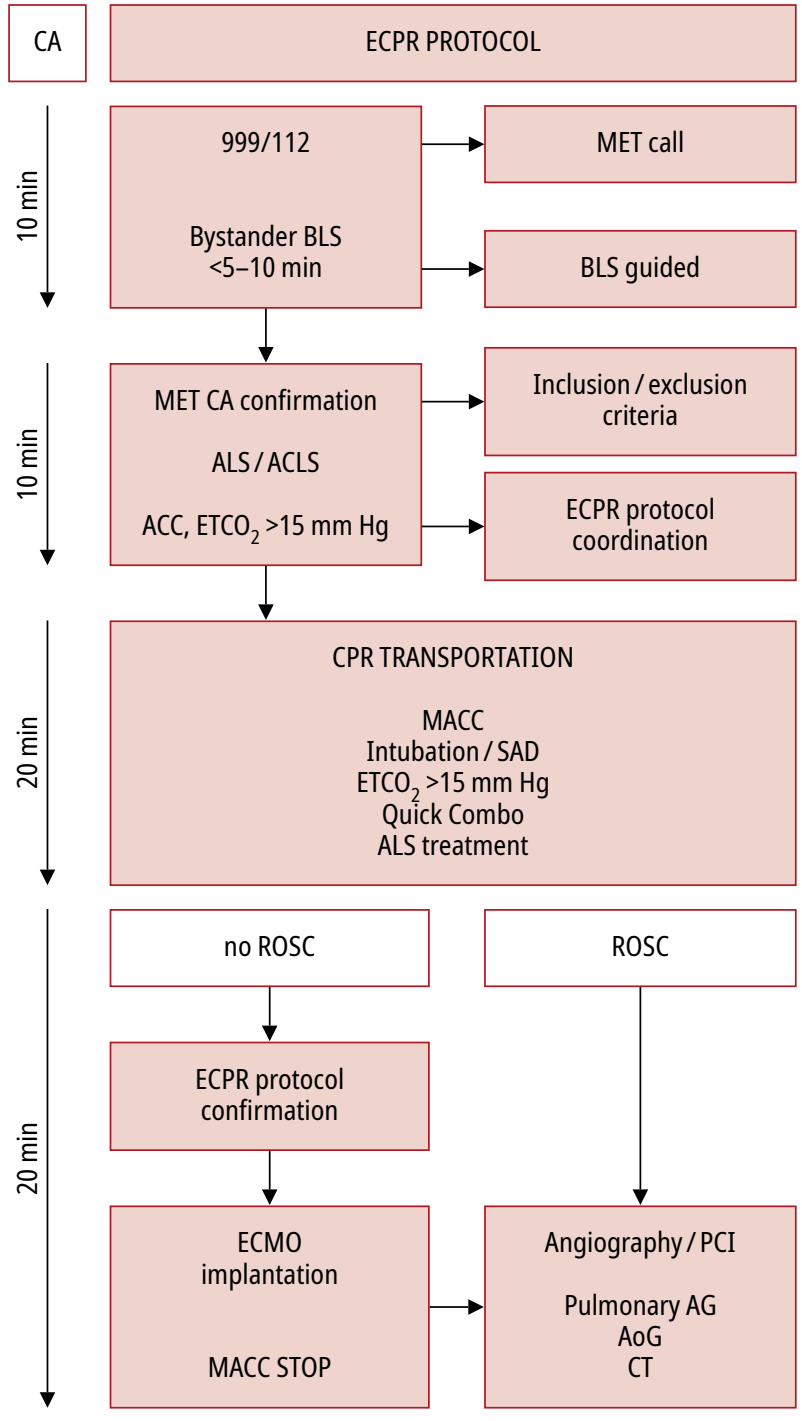

FIGURE 2 The algorithm for the Pilot POHCA (Poznan Out-of-Hospital Cardiac Arrest) Program Abbreviations: MACC, mechanical automated chest compression; AG, angiography; ALS / ACLS, advanced life support/ advanced cardiovascular life support; AoG, aortography; BLS, basic life support; CA, cardiac arrest; CT, computed tomography; ECMO, extracorporeal membrane oxygenation; ECPR, extended cardiopulmonary resuscitation; $\mathrm{ETCO}_{2}$, end-tidal carbon dioxide; MET, medical emergency team; PCI, percutaneous coronary intervention; SAD, supraglottic airway devices; ROSC, return of spontaneous circulation

analysis, we adopted a range of 18 to 65 years which appears to be the optimal for the initiation phase of ECPR procedure implementation in the Poznań region.

The survival rate following ECPR is also determined by the time between the onset of resuscitation measures and the initiation of ECMO support, which is defined as no-flow time. During this period, perfusion of organs is impaired which can lead to multiorgan failure and cerebral ischemia. ${ }^{34}$ This is confirmed by data from in-hospital cardiac arrest cases, where the survival rate was $50 \%$ at low-flow time of less than 30 minutes, $30 \%$ between 30 to 60 minutes, and only $18 \%$ when it exceeded 1 hour. ${ }^{35}$ OHCA is usually considered as refractory to CPR after
10 to 30 minutes of CPR/ALS (country-related variability), after which the decision to implement ECPR is made. $7,31,36$ Taking into account the results of available studies, to reach the optimal outcome, the duration of CPR should be up to 10 minutes to identify persistent OHCA, and the time from the site of SCA to the hospital door should not exceed 40 minutes. Introduction of an ECMO device usually takes 15 to 20 minutes, thus, a total OHCA-to-ECMO start time should be up to 60 minutes.

Based on the experience of our ECMO Team of the ECMO for Greater Poland project, we estimate that 20 minutes are enough to prepare a catheterization laboratory with an angiograph, to complete priming of the ECMO device, and to perform percutaneous cannulation of peripheral vessels (with the Seldinger technique). The intervening cardiologists play a crucial role and their skills are helpful during cannulation and are mandatory to carry out the cause-directed therapeutic procedures during extracorporeal support. We proposed an algorithm for the pilot ECPR program in Poznan (Poznan Out-ofHospital Cardiac Arrest, POHCA) and prepared it for implementation (FIGURE2). ${ }^{14}$

The principal conditions for the effectiveness of ECPR and optimal survival rate after OHCA are high-quality chest compressions and ventilation with minimal breaks. ${ }^{33,37}$ A sensible alternative to manual chest compressions is the MACC device. ${ }^{38}$ It can be used at the site of emergency, during transfer to the hospital, but also in catheterization laboratories during preparation for ECMO implantation. It was possible to transport 26 patients with recurrence of SCA after ROSC noted during ALS (nonpermanent ROSC) to the hospital thanks to a MACC device.

Paramedics or SCA witnesses should follow the valid recommendations to minimize, if necessary, breaks in CPR including for ventilation, defibrillation, or other life-saving maneuvers. Chest compression fraction, defined as the time actually spent on chest compressions during CPR, aims to minimize these breaks. ${ }^{11}$ Achieving a chest compression fraction of at least $60 \%$ and striving for $80 \%$ significantly improves chances of survival. ${ }^{39,40}$ Another device for assessing chest compression quality is also available. Continuous monitoring of $\mathrm{CO}_{2}$ in exhaled air $\left(\mathrm{ETCO}_{2}\right)$ provides information not only on the quality of chest compressions but also confirms proper airway management and ventilation, and thus allows to estimate the potential for ROSC. ${ }^{41}$ The amount of $\mathrm{CO}_{2}$ in the exhaled air is determined by the cardiac output and maintaining an $\mathrm{ETCO}_{2}$ value of more than $15 \mathrm{~mm} \mathrm{Hg}$ is associated with significantly higher probability of ROSC..$^{41}$ Of note, most of EMS ambulances, particularly in larger Polish agglomerations, are equipped with MACC device and capnography to assess $\mathrm{ETCO}_{2}$. 
Limitations This retrospective study has some substantial limitations. The main limitation is that mainly the medical charts of EMS were analyzed, thus confining the study predominantly to the pre-hospital part of management of patients with OHCA. Our assessment of further outcomes is based on the incomplete in-hospital clinical data and may be associated with marked bias. However, in spite of these obvious limitation, our study demonstrated the weakest points in the management of patients with OHCA as well as lend support to the implementation of ECPR as a part of a pioneer Polish regional OHCA Program. We cannot exclude that substantial improvement in the education of Polish society with respect to SCA and BLS may increase the number of patients surviving OHCA and potential candidates for ECMO support prior to the life-saving cardiovascular interventions.

Conclusions Our analysis showed that in some patients after OHCA (due to potentially reversible causes), it would have been possible to implement ECPR as a crucial part of the OHCA Program, and as a result, probably to improve early outcomes of these patients.

\section{ARTICLE INFORMATION}

\section{CONFLICT OF INTEREST None declared.}

OPEN ACCESS This is an Open Access article distributed under the terms of the Creative Commons Attribution-NonCommercial-NoDerivatives 4.0 International License (CC BY-NC-ND 4.0), allowing third parties to download articles and share them with others, provided the original work is properly cited, not changed in any way, distributed under the same license, and used for noncommercial purposes only. For commercial use, please contact the journal office at kardiologiapolska@ptkardio.pl.

HOW To CITE Sip M, Puślecki M, Kłosiewicz T, et al. A concept for the development of a pioneer regional Out-of-Hospital Cardiac Arrest Program to improve patient outcomes. Kardiol Pol. 2020; 78: 875-881. doi:10.33963/KP.15433

\section{REFERENCES}

1 Information on socio-economic situation of the country in 2018. Statistics Poland. https://stat.gov.pl/en/topics/statistical-yearbooks/statistical-yearbooks/ demographic-yearbook-of-poland-2018,3,12.html. Accessed March 1, 2020.

2 Wojtyniak B, Goryński P. Health situation of the Polish population and its determinants - synthesis. National Institute of Public Health - National Institute of Hygiene: 2018. Warsaw, Poland: 492.

3 Monsieurs KG, Nolan JP, Bossaert LL, et al. European Resuscitation Council guidelines for resuscitation 2015. Resuscitation. 2015; 95: 1-80.

4 Berdowski J, Berg RA, Tijssen JG, Koster RW. Global incidences of out-of-hospital cardiac arrest and survival rates: systematic review of 67 prospective studies. Resuscitation. 2010; 81: 1479-1487.

5 Cebula GM, Osadnik S, Wysocki M, et al. Comparison of the early effects of out-of-hospital resuscitation in selected urban and rural areas in Poland. A preliminary report from the Polish Cardiac Arrest Registry by the Polish Resuscitation Council. Kardiol Pol. 2016; 74: 356-361.

6 Nadolny K, Zyśko D, Obremska M, et al. Analysis of out-of-hospital cardiac arrest in Poland in a 1-year period: data from the POL-OHCA registry. Kardiol Pol. 2020; 78: 404-411.

7 Grunau B, Reynolds J, Scheuermeyer F, et al. Relationship between time-to-ROSC and survival in out-of-hospital cardiac arrest ECPR candidates: when is the best time to consider transport to hospital? Prehosp Emerg Care. 2016; 20: $615-622$

8 Reynolds JC, Grunau BE, Elmer J, et al. Prevalence, natural history, and time-dependent outcomes of a multi-center north American cohort of out-of-hospital cardiac arrest extracorporeal CPR candidates. Resuscitation. 2017; 117: 24-31.

9 Kuroki N, Abe D, Iwama T, et al. Association between delay to coronary reperfusion and outcome in patients with acute coronary syndrome undergoing extracorporeal cardiopulmonary resuscitation. Resuscitation. 2017; 114: 1-6.
10 Link MS, Berkow LC, Kudenchuk PJ, et al. Part 7: Adult advanced cardiovascular life support: 2015 American Heart Association guidelines update for cardiopulmonary resuscitation and emergency cardiovascular care. Circulation. 2015; 132 (suppl 2): S444-S464.

11 Haas NL, Coute RA, Hsu CH, et al. Descriptive analysis of extracorporeal cardiopulmonary resuscitation following out-of-hospital cardiac arrest - an ELSO registry study. Resuscitation. 2017; 119: 56-62.

12 Puślecki M, Sip M, Ligowski M, et al. Poznan Out-of-Hospital Cardiac Arrest (POHCA) study - the first Polish ECPR pilot trial protocol. Perfusion. 2019; 34 (suppl. 1): P01-P068, 122.

13 Puślecki M; Ligowski M, Stefaniak S, et al. "Extracorporeal Membrane Oxygenation for Greater Poland" program: how to save lives and develop organ donation? Transplant Proc. 2018; 50: 1957-1961.

14 Puślecki $M$, Ligowski $M$, Stefaniak $S$, et al. Using simulation to create a unique regional ECMO program for the Greater Poland region. Qatar Med J. 2017; 2017: 79.

15 Puślecki M, Ligowski M, Dąbrowski M, et al. BEST Life - "Bringing ECMO simulation to life" - how medical simulation improved a regional ECMO program. Artif Organs. 2018; 42: 1052-1061.

16 Belohlavek J, Kucera K, Jarkovsky J, et al. Hyperinvasive approach to out-of hospital cardiac arrest using mechanical chest compression device, prehospital intraarrest cooling, extracorporeal life support and early invasive assessment compared to standard of care. A randomized parallel groups comparative study proposal. "Prague OHCA study." J Transl Med. 2012; 10: 163.

17 Abrams D, Garan AR, Abdelbary A, et al.; International ECMO Network (ECMONet) and the Extracorporeal Life Support Organization (ELSO). Position paper for the organization of ECMO programs for cardiac failure in adults. Intensive Care Med. 2018; 44: 717-729.

18 Puślecki $M$, Ligowski $M$, Dąbrowski $M$, et al. Unique regional program "ECMO for Greater Poland." Eur J Heart Fail. 2017: 19 (suppl 2): 46-47.

19 Analysis of resuscitation activities undertaken in SCA cases occurring in non-hospital settings. Katowice, Poland. http://www.eioba.pl/a/2ty3/jak-uratowac-ludzkie-zycie-czyli-zwyczajni-niezwyczajni-w-akcji. Accessed March 1, 2020.

20 Gach D, Nowak JU, Krzych Łl. Epidemiology of out-of-hospital cardiac arrest in the Bielsko-Biala district: a 12-month analysis. Kardiol Pol. 2016; 74: 1180-1187.

21 Sielski J, Kaziród-Wolski K, Solnica M, et al. Outofhospital cardiac arrest registry: preliminary results from the Świętokrzyskie Province. Kardiol Pol. 2019; 77: 710-712.

22 Woźniak K, Jędrzejczyk M, Dorota 0, Lewandowski B. Knowledge of students on providing premedical first aid. Journal of Education, Health and Sport. 2017; 7: 1101-1112.

23 Kozłowski D, Kłosiewicz T, Kowalczyk A, et al. The knowledge of public access to defibrillation in selected cities in Poland. Arch Med Sci. 2013; 9: 27-33.

24 Culley LL, Rea TD, Murray JA, et al. Public access defibrillation in outof-hospital cardiac arrest: a community-based study. Circulation. 2004; 109: 1859-1863.

25 Pogorzelczyk K, Bandurska E, Łopaciński T, et al. Tri-city inhabitants' state of knowledge on automated external defibrillator and their readiness to use it. Hygeia Public Health. 2018; 53: 178-184.

26 Tanguay-Rioux X, Grunau B, Neumar R, et al. Is initial rhythm in OHCA a predictor of preceding no flow time? Implications for bystander response and ECPR candidacy evaluation. Resuscitation. 2018; 128: 88-92.

27 Jeong W], Lee JW, Yoo YH, et al. Extracorporeal cardiopulmonary resuscitation in bedside echocardiography-diagnosed massive pulmonary embolism. Am J Emerg Med. 2015; 33: 1545.e1-2.

28 Morita S, Inokuchi S, Yamagiwa T, et al. Efficacy of portable and percutnaeous cardiopulmonary bypass rewarming versus that of conventional internal rewarming for patients with accidental deep hypothermia. Crit Care Med. 2011; 39: 1064-1068.

29 Nakashima T, Noguchi T, Tahara Y, et al. Patients with refractory out-of-cardiac arrest and sustained ventricular fibrillation as candidates for extracorporeal cardiopulmonary resuscitation. Circ J. 2019; 83: 1011-1018.

30 Andersen LW, Bivens MJ, Giberson T, et al. The relationship between age and outcome in out-of-hospital cardiac arrest patients. Resuscitation. 2015; 94: 49-54. 31 Chen YS, Lin JW, Yu HY, et al. Cardiopulmonary resuscitation with assisted extracorporeal life-support versus conventional cardiopulmonary resuscitation in adults with in-hospital cardiac arrest: an observational study and propensity analysis. Lancet. 2008; 372: 554-561.

32 Lamhaut L, Hutin A, Puymirat E, et al. A pre-hospital extracorporeal cardio pulmonary resuscitation (ECPR) strategy for treatment of refractory out hospital cardiac arrest: an observational study and propensity analysis. Resuscitation. 2017; 117: 109-117.

33 Stub D, Bernard S, Pellegrino V, et al. Refractory cardiac arrest treated with mechanical CPR, hypothermia, ECMO and early reperfusion (the CHEER trial). Resuscitation. 2015; 86: 88-94

34 Debaty G, Babaz V, Durand M, et al. Prognostic factors for extracorporeal cardiopulmonary resuscitation recipients following out-of-hospital refractory cardiac arrest. A systematic review and meta-analysis. Resuscitation. 2017; 112: 1-10. 35 Fagnoul D, Combes A, De Backer D. Extracorporeal cardiopulmonary resuscitation. Curr Opin Crit Care. 2014; 20: 259-265. 
36 Resuscitation guidelines 2015. https://www.resus.org.uk/resuscitation-guidelines/prehospital-resuscitation/. Accessed March 1, 2020.

37 Soar J, Nolan J.P, Böttiger B.W, et al. European Resuscitation Council Guidelines for Resuscitation 2015: section 3. Adult advanced life support. Resuscitation. 2015; 95: 100-147.

38 Wang PL, Brooks SC. Mechanical versus manual chest compressions for cardiac arrest. Cochrane Database Syst Rev. 2018; 8: CD007260.

39 Vaillancourt C, Everson-Stewart S, Christenson J, et al. The impact of increased chest compression fraction on return of spontaneous circulation for out-of-hospital cardiac arrest patients not in ventricular fibrillation. Resuscitation. 2011; 82: 1501-1507.

40 Meaney PA, Bobrow BJ, Mancini ME, et al. Cardiopulmonary resuscitation quality: Improving cardiac resuscitation outcomes both inside and outside the hospital: a consensus statement from the American Heart Association. Circulation. 2013; 128: 417-435.

41 Kłosiewicz T. Capnometry as a device helpful in resuscitation [in Polish]. BiTP. Bezpieczeństwo i Technika Pożarnicza. 2016; 42: 203-208. 